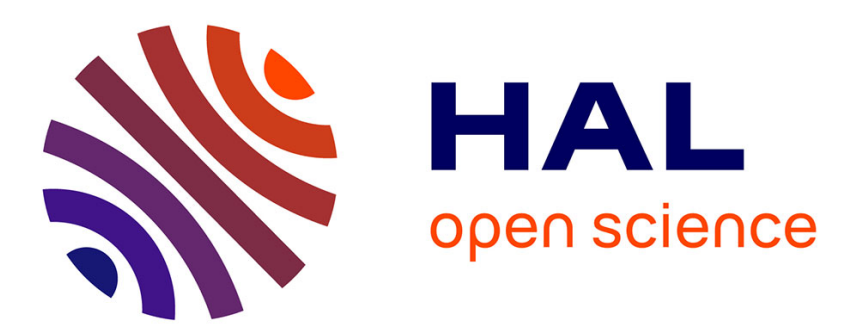

\title{
Optimal analysis-aware parameterization of computational domain in 3D isogeometric analysis
}

Gang Xu, Bernard Mourrain, Régis Duvigneau, André Galligo

\section{To cite this version:}

Gang Xu, Bernard Mourrain, Régis Duvigneau, André Galligo. Optimal analysis-aware parameterization of computational domain in 3D isogeometric analysis. Computer-Aided Design, 2013, 45 (4), 10.1016/j.cad.2011.05.007 . inria-00539616

\section{HAL Id: inria-00539616 \\ https://hal.inria.fr/inria-00539616}

Submitted on 24 Nov 2010

HAL is a multi-disciplinary open access archive for the deposit and dissemination of scientific research documents, whether they are published or not. The documents may come from teaching and research institutions in France or abroad, or from public or private research centers.
L'archive ouverte pluridisciplinaire HAL, est destinée au dépôt et à la diffusion de documents scientifiques de niveau recherche, publiés ou non, émanant des établissements d'enseignement et de recherche français ou étrangers, des laboratoires publics ou privés. 


\title{
Optimal analysis-aware parameterization of computational domain in 3D isogeometric analysis
}

\author{
Gang $\mathrm{Xu}^{\mathrm{a}}$, Bernard Mourrain ${ }^{\mathrm{a}}$, Régis Duvigneau ${ }^{\mathrm{b}}$, André Galligo $^{\mathrm{c}}$ \\ ${ }^{a}$ Galaad, INRIA Sophia-Antipolis, 2004 Route des Lucioles, 06902 Cedex, France \\ ${ }^{b}$ OPALE, INRIA Sophia-Antipolis, 2004 Route des Lucioles, 06902 Cedex, France \\ ${ }^{c}$ University of Nice Sophia-Antipolis, 06108 Nice Cedex 02, France
}

\begin{abstract}
In isogeometric analysis framework, computational domain is exactly described using the same representation as that employed in the CAD process. For a CAD object, we can construct various computational domain with same shape but with different parameterization. One basic requirement is that the resulting parameterization should have no self-intersections. In this paper, a linear and easy-to-check sufficient condition for injectivity of trivariate B-spline parameterization is proposed. By an example of 3D thermal conduction problem, we show that different parameterization of computational domain has different impact on the simulation result and efficiency in isogeometric analysis. For problems with exact solutions, we propose a shape optimization method to obtain optimal parameterization of computational domain. The proposed injective condition is used to check the injectivity of initial trivariate B-spline parameterization constructed by discrete Coons volume method, which is the generalization of discrete Coons patch method. Several examples and comparisons are presented to show the effectiveness of the proposed method. Compared with the initial parameterization during refinement, the optimal parameterization can achieve the same accuracy but with less degrees of freedom.
\end{abstract}

Keywords: isogeometric analysis; analysis-aware parameterization of computational domain; injectivity; shape optimization; steepest descent method

Email addresses: Gang.Xu@inria.fr (Gang Xu), Bernard.Mourrain@inria.fr (Bernard Mourrain ), Regis.Duvigneau@inria.fr (Régis Duvigneau), galligo@unice.fr (André Galligo) 


\section{Introduction}

CAD modeling software usually relies on splines or NURBS representations, but the analysis software for CAD object uses mesh-based geometric descriptions (structured or unstructured). Therefore, in conventional approaches, several information transfers occur during the design phase, yielding approximations and non-linear transformations that can significantly deteriorate the overall efficiency of the design optimization procedure.

The isogeometric analysis (IGA for short) approach proposed by Hughes et al. [19] is employed to overcome this difficulty by using CAD standards as unique representation for all disciplines. For $3 \mathrm{D}$ analysis problems, the isogeometric approach consists in developing methods that use NURBS representations for all design and analysis tasks:

- the geometry is defined by NURBS surfaces;

- the computation domain is defined by trivariate NURBS volumes instead of discrete meshes;

- the solution fields are obtained by using a finite-element approach that uses NURBS basis functions instead of classical Lagrange polynomials;

- the optimizer controls directly NURBS control points.

This framework allows to compute the analysis solution on the exact geometry (not a discretized geometry), obtain a more accurate solution (high-order approximation), reduce spurious numerical sources of noise that deteriorate convergence, avoid data transfers between the design and analysis phases. Moreover, NURBS representation is naturally hierarchical and allows to perform refinement operations to improve the analysis result.

In finite element analysis (FEA), mesh generation, which generates discrete geometry as computational domain from given CAD object, is a key and the most time-consuming step. In IGA framework, parameterization of computational domain, which corresponds to the mesh generation in FEA, also has some impact on analysis result and efficiency. Moreover, in FEA, one can perform arbitrary refinements on the computational mesh, but in IGA using tensor product B-splines, the refinement is not arbitrary, we can only perform refinement operations in $u$ direction and $v$ direction by knot insertion or degree evaluation. Hence, parameterization of computational domain is more important in IGA. 
The parameterization of a computational domain in IGA is determined by control points, knot vectors and the degrees of B-spline objects. For IGA problem of three dimension, the knot vectors and the degree of computational domain are determined by the given boundary surfaces. Hence, finding the optimal placement of inner control points for a specified physical problem, is a key issue in IGA. A basic requirement of resulting parameterization for IGA is that it doesn't have self-intersections. In this paper, we first propose a linear and easy-to-test sufficient condition for injectivity of trivariate B-spline parameterization. Then we show that different parameterizations of computational domain has different impact on the simulation results in IGA. For problems with exact solutions, a shape optimization method is proposed to obtain an optimal parameterization of computational domain. Discrete Coons volume method is proposed to construct initial trivariate B-spline parameterization from given boundary surfaces. Some examples and comparisons are presented based on the heat conduction problem to show the effectiveness of the proposed method.

The remainder of the paper is organized as follows. Section 2 reviews the related work in isogeometric analysis. Section 3 proposes the linear sufficient conditions for injectivity of trivariate B-spline parameterization. Section 4 describes a test IGA model and shows the impact of different parameterizations of computational domain. Section 5 presents the discrete Coons volume method to construct initial trivariate B-spline parameterization from boundary surfaces, and the shape optimization method to obtain an optimal parameterization of a computational domain. Some examples and comparisons are also presented in Section 5. Finally, we conclude this paper and outline future works in Section 6.

\section{Related work}

In this section, we review related works in IGA and parameterization of computational domains.

The concept of IGA was firstly proposed by T.R Hughes et al. [19] in 2005 to achieve the seamless integration of CAD and FEA. Since then, many researchers in the fields of mechanical engineering and geometric modeling were involved in this topic. The current work on isogeometric analysis can be classified into three categories: (1) application of IGA to various simulation problems $[2,5,6,11,14,18,20,29,31]$; (2) application of various geometric modeling tools to IGA $[7,13,24,8]$; (3) accuracy and efficiency improvement of IGA framework by reparameterization and refinement operations [1, 3, 9, 10, 15, 21, 25]. 
The topic of this paper belongs to the third field. As far as we know, there are few works on the parametrizations of computational domains for IGA. T. Martin et al. [25] proposed a method to fit a genus-0 triangular mesh by B-spline volume parameterization, based on discrete volumetric harmonic functions; this can be used to build computational domains for 3D IGA problems. A variational approach for constructing NURBS parameterization of swept volumes is proposed by M. Aigner et al [1]. Many free-form shapes in CAD systems, such as blades of turbines and propellers, are covered by this kind of volumes. E. Cohen et.al. [9] proposed the concept of analysis-aware modeling, in which the parameters of CAD models should be selected to facilitate isogeometric analysis. Approximate implicitization technique is used for parametrization of computational domain in [26]. In [30], we consider optimal parametrisation of planar domains for $2 \mathrm{D}$ isogeometric analysis. In this paper, we extend this approach to volumetric problems. A method for generating optimal analysis-aware parameterization of computational domain for 3D IGA problems is proposed based on shape optimization method.

\section{A linear sufficient condition for injectivity of planar B-spline parameterization}

The main idea of the isogeometric approach is to use the same representation for the geometry and the physical solutions we are interested in. Schematically, the geometry $\Omega$ involved in the physical problem can be a parametric volume in a three-dimensional space $\mathbb{R}^{3}$. Let us call $\mathbf{x}=$ $(x, y, z)$ the coordinates associated to this space. In our case, this geometry will be represented by a parameterization $\sigma$ for a domain $\mathcal{P}$ of the parameter space. Let us call $\mathbf{u}$ the coordinates of this parameter domain, which could be of dimension 3 for a volume. This parameterization will be given by B-spline functions with knots in $\mathcal{P}$ and control points in $\mathbb{R}^{3}$.

The concept of isogeometry consists in representing the physical quantities $\Phi \in \mathbb{R}^{p}$ on the geometry $\Omega$ using the same type of B-spline representation as for the geometry $\Omega$. In other words, given a point $\mathbf{x}=\sigma(\mathbf{u}) \in \Omega$ with $\mathbf{u} \in \mathcal{P}$, we associate to it the physical quantities $\Phi(\mathbf{u})$ where $\Phi(\mathbf{u})$ is a B-spline function with nodes in $\mathcal{P}$ and control points in $\mathbb{R}^{p}$. This means that the map $\mathbf{x} \in \Omega \mapsto \Phi \in \mathbb{R}^{p}$ is defined implicitly as $\mathbf{x} \mapsto \Phi \circ \sigma^{-1}(\mathbf{x})$.

Consequently, the framework of isogeometry is thus valid when the parameterization $\sigma$ of the geometry is injective (or bijective on its image). We are going to describe sufficient and easy-tocheck conditions for the injectivity of $\sigma$. We will consider this problem in the context of finding 
a "good" parameterization of a domain when its boundary is given. In [23], a general sufficient condition is proposed for injective parameterization.

Proposition 1. Suppose that $\sigma$ is a $C^{1}$ parameterization from a compact domain $\mathcal{P} \subset \mathbb{R}^{n}$ with a connected boundary to a geometry $\Omega \subset \mathbb{R}^{n}$. If $\sigma$ is injective on the boundary $\partial \mathcal{P}$ of $\mathcal{P}$ and its Jacobian $J_{\sigma}$ does not vanish on $\mathcal{P}$, then $\sigma$ is injective.

For a parameterization $\sigma$ from $[a, b] \times[c, d] \times[e, f]$ to $\Omega \subset \mathbb{R}^{3}$, we define the boundary surfaces as the image of $\{a\} \times[c, d] \times[e, f],\{b\} \times[c, d] \times[e, f],[a, b] \times[c, d] \times\{e\},[a, b] \times[c, d] \times\{f\},[a, b] \times[e, f] \times\{c\}$, $[a, b] \times[e, f] \times\{d\}$, by $\sigma$. We say that $\sigma$ defines a regular boundary if these surfaces do not intersect pairwise, except at their end curves and if they have no self-intersection.

As a consequence of the previous proposition, we get the following injectivity test for standard trivariate B-spline parameterization of a 3D computational domain.

Proposition 2. Let $\sigma$ be a $C^{1}$ parameterization from $[a, b] \times[c, d] \times[e, f]$ to $\Omega \subset \mathbb{R}^{3}$ which defines a regular boundary. If its Jacobian $J_{\sigma}$ does not vanish on $[a, b] \times[c, d] \times[e, f]$, then $\sigma$ is injective.

These tests involve injectivity conditions on the boundary, which can be checked recursively using the same techniques, non-intersection tests for boundary curves and surfaces which are provided for instance by geometric (subdivision) algorithms and the local injectivity condition corresponding to the non-vanishing of the Jacobian. This last condition requires to test on all the domain $\Omega$ that the Jacobian does not vanish. Hereafter we propose a sufficient and easy-to-test condition to ensure the local injectivity condition.

We consider the case of a trivariate parameterization

$$
\sigma: \mathbf{u} \in \mathcal{P}:=[a, b] \times[c, d] \times[e, f] \mapsto \sigma(\mathbf{u}):=\sum_{0 \leq i \leq l_{1}, 0 \leq, j \leq l_{2}, 0 \leq k \leq l_{3}} \mathbf{c}_{i, j, k} N_{i, j, k}(\mathbf{u}),
$$

where $\mathbf{c}_{i, j, k} \in \mathbb{R}^{3}$ are the control points and $N_{i, j, k}(\mathbf{u})$ are the B-spline basis functions. The derivative of $\sigma(\mathbf{u})$ with respect to $\mathbf{u}_{1}$ can be expressed in terms of the differences $\Delta_{i, j, k}^{1}:=\mathbf{c}_{i+1, j, k}-\mathbf{c}_{i, j, k}$ :

$$
\partial_{u_{1}} \sigma(\mathbf{u}):=\sum_{0 \leq i \leq l_{1}-1,0 \leq j \leq l_{2}, 0 \leq k \leq l_{3}} \omega_{i, j, k}^{1} \Delta_{i, j, k}^{1} N_{i, j, k}^{1}(\mathbf{u}),
$$

where $N_{i, j, k}^{1}$ is the B-spline basis function with one degree less in $\mathbf{u}_{1}, \omega_{i, j, k}^{1}$ is a positive factor. We denote by $\mathcal{C}_{1}(\mathbf{c})$ the convex cone of $\mathbb{R}^{3}$ generated by the half rays $\mathbb{R}_{+} \cdot \frac{\Delta_{i, j, k}^{1}}{\left\|\Delta_{i, j, k}^{1}\right\|}$. 
Similarly, the derivative of $\sigma(\mathbf{u})$ with respect to $\mathbf{u}_{2}$ can be expressed in terms of the differences $\Delta_{i, j, k}^{2}:=\mathbf{c}_{i, j+1, k}-\mathbf{c}_{i, j, k}:$

$$
\partial_{u_{2}} \sigma(\mathbf{u}):=\sum_{0 \leq i \leq l_{1}, 0 \leq j \leq l_{2}-1,0 \leq k \leq l_{3}} \omega_{i, j, k}^{2} \Delta_{i, j, k}^{2} N_{i, j, k}^{2}(\mathbf{u}),
$$

where $N_{i, j, k}^{2}$ is the B-spline basis with one degree less in $\mathbf{u}_{2}, \omega_{i, j, k}^{2}$ is a positive factor. We denote by $\mathcal{C}_{2}(\mathbf{c})$ the convex cone of $\mathbb{R}^{3}$ generated by the half rays $\mathbb{R}_{+} \cdot \frac{\Delta_{i, j, k}^{2}}{\left\|\Delta_{i, j, k}^{2}\right\|}$.

Similarly, the derivative of $\sigma(\mathbf{u})$ with respect to $\mathbf{u}_{3}$ can be expressed in terms of the differences $\Delta_{i, j, k}^{3}:=\mathbf{c}_{i, j, k+1}-\mathbf{c}_{i, j, k}:$

$$
\partial_{u_{3}} \sigma(\mathbf{u}):=\sum_{0 \leq i \leq l_{1}, 0 \leq j \leq l_{2}, 0 \leq k \leq l_{3}-1} \omega_{i, j, k}^{3} \Delta_{i, j, k}^{3} N_{i, j, k}^{3}(\mathbf{u}),
$$

where $N_{i, j, k}^{3}$ is the B-spline basis with one degree less in $\mathbf{u}_{3}, \omega_{i, j, k}^{3}$ is a positive factor. We denote by $\mathcal{C}_{3}(\mathbf{c})$ the convex cone of $\mathbb{R}^{3}$ generated by the half rays $\mathbb{R}_{+} \cdot \frac{\Delta_{i, j, k}^{3}}{\left\|\Delta_{i, j, k}^{3}\right\|}$. If this cone is generated by two opposite vectors, which are on a straight line, we define $\mathcal{C}_{i}(\mathbf{c})$ as any half-space. For a cone $\mathcal{C}$, we denote by $\mathcal{C}^{*}$ the cone without the origin 0 .

We say that two cones $\mathcal{C}_{1}, \mathcal{C}_{2}$ are transverse if $\mathbb{R} \cdot \mathcal{C}_{1}$ and $\mathbb{R} \cdot \mathcal{C}_{2}$ intersect only at $\{0\}$. We say that three cones $\mathcal{C}_{1}, \mathcal{C}_{2}, \mathcal{C}_{3}$ are cotransverse if

- 0 is a vertex of the convex hull of $\mathcal{C}_{1}, \mathcal{C}_{2}, \mathcal{C}_{3}$;

- the convex hull of the cones $\mathcal{C}_{i}, \mathcal{C}_{j}$ and the cone $\mathcal{C}_{k}$ are transverse for all $\{i, j, k\}=\{1,2,3\}$.

Notice in particular that if $\mathcal{C}_{1}, \mathcal{C}_{2}, \mathcal{C}_{3}$ are cotransverse, then $\mathcal{C}_{i}, \mathcal{C}_{j}$ are transverse for $1 \leq i \neq j \leq 3$. This definition implies the following result:

Lemma 1. If three convex cones $\mathcal{C}_{1}, \mathcal{C}_{2}, \mathcal{C}_{3}$ are cotransverse, then $\left|\mathbf{u}_{1}, \mathbf{u}_{2}, \mathbf{u}_{3}\right|$ has a constant sign for all $\mathbf{u}_{1} \in \mathcal{C}_{1}^{*}, \mathbf{u}_{2} \in \mathcal{C}_{2}^{*}, \mathbf{u}_{3} \in \mathcal{C}_{3}^{*}$.

Proof. As the cones $\mathcal{C}_{1}, \mathcal{C}_{2}, \mathcal{C}_{3}$ are convex, the set $\mathcal{C}_{1}^{*}, \mathcal{C}_{2}^{*}, \mathcal{C}_{3}^{*}$ are connected. Thus the lemma is proved if we can show that $\left|\mathbf{u}_{1}, \mathbf{u}_{2}, \mathbf{u}_{3}\right| \neq 0$ for all $\mathbf{u}_{1} \in \mathcal{C}_{1}^{*}, \mathbf{u}_{2} \in \mathcal{C}_{2}^{*}, \mathbf{u}_{3} \in \mathcal{C}_{3}^{*}$.

As 0 is a vertex of the convex hull of $\mathcal{C}_{1}, \mathcal{C}_{2}, \mathcal{C}_{3}$, there exists a plane $H_{0}$ such that $\forall \mathbf{u} \in \mathcal{C}_{i}^{*}$ $i=1,2,3, H_{0}(\mathbf{u})>0$.

As the convex hull of the cones $\mathcal{C}_{i}, \mathcal{C}_{j}$ and the cone $\mathcal{C}_{k}$ are transverse for all $\{i, j, k\}=\{1,2,3\}$, there exist a plane $H_{k}$ such that $\forall \mathbf{u} \in \mathcal{C}_{i}^{*} \cup \mathcal{C}_{j}^{*}, H_{k}(\mathbf{u})>0$ and $\forall \mathbf{u} \in \mathcal{C}_{k}^{*}, H_{k}(\mathbf{u})<0$. 
Suppose that there exists $\mathbf{u}_{1} \in \mathcal{C}_{1}^{*}, \mathbf{u}_{2} \in \mathcal{C}_{2}^{*}, \mathbf{u}_{3} \in \mathcal{C}_{3}^{*}$ with $\left|\mathbf{u}_{1}, \mathbf{u}_{2}, \mathbf{u}_{3}\right|=0$. Then there exists $\alpha_{1}, \alpha_{2}, \alpha_{3} \in \mathbb{R}$ not all zero, such that $\alpha_{1} \mathbf{u}_{1}+\alpha_{2} \mathbf{u}_{2}+\alpha_{3} \mathbf{u}_{3}=0$.

If one of the $\alpha_{i}$ is zero, then the cones $\mathcal{C}_{j}$ and $\mathcal{C}_{k}$ (for $\left.\{i, j, k\}=\{1,2,3\}\right)$ cannot be transverse, which is excluded.

If all $\alpha_{i}$ have the same sign (say $\alpha_{i}>0$ ), then

$$
H_{0}\left(\alpha_{1} \mathbf{u}_{1}+\alpha_{2} \mathbf{u}_{2}+\alpha_{3} \mathbf{u}_{3}\right)=\alpha_{1} H_{0}\left(\mathbf{u}_{1}\right)+\alpha_{2} H_{0}\left(\mathbf{u}_{2}\right)+\alpha_{3} H_{0}\left(\mathbf{u}_{3}\right)>0
$$

This is not possible if $\alpha_{1} \mathbf{u}_{1}+\alpha_{2} \mathbf{u}_{2}+\alpha_{3} \mathbf{u}_{3}=0$.

If the $\alpha_{i}$ have not the same sign, then one has a sign ( say $\alpha_{i}<0$ ) and the two other have the opposite sign $\left(\alpha_{j}>0, \alpha_{k}>0\right.$ for $\left.\{i, j, k\}=\{1,2,3\}\right)$, then

$$
H_{i}\left(\alpha_{i} \mathbf{u}_{i}+\alpha_{j} \mathbf{u}_{j}+\alpha_{k} \mathbf{u}_{k}\right)=\alpha_{i} H_{i}\left(\mathbf{u}_{i}\right)+\alpha_{j} H\left(\mathbf{u}_{j}\right)+\alpha_{k} H_{i}\left(\mathbf{u}_{k}\right)>0 .
$$

This is not possible if $\alpha_{1} \mathbf{u}_{1}+\alpha_{2} \mathbf{u}_{2}+\alpha_{3} \mathbf{u}_{3}=0$.

We deduce that for all $\mathbf{u}_{1} \in \mathcal{C}_{1}^{*}, \mathbf{u}_{2} \in \mathcal{C}_{2}^{*}, \mathbf{u}_{3} \in \mathcal{C}_{3}^{*}$, the vectors $\mathbf{u}_{1}, \mathbf{u}_{2}, \mathbf{u}_{3}$ are linearly independent and $\left|\mathbf{u}_{1}, \mathbf{u}_{2}, \mathbf{u}_{3}\right| \neq 0$.

Proposition 3. Let $\sigma$ be a trivariate B-spline parametrisation, which is at least $C^{1}$ from $\mathcal{P}:=$ $[a, b] \times[c, d] \times[e, f]$ to $\Omega \subset \mathbb{R}^{3}$ given by the control points $\mathbf{c}$. If the boundary surfaces do not intersect and have no self-intersection point and the cones $\mathcal{C}_{1}(\mathbf{c}), \mathcal{C}_{2}(\mathbf{c}), \mathcal{C}_{3}(\mathbf{c})$ are transverse, then $\sigma$ is injective on $\mathcal{P}$.

Proof. We check first that the transversality of the cones $\mathcal{C}_{1}(\mathbf{c}), \mathcal{C}_{2}(\mathbf{c}), \mathcal{C}_{3}(\mathbf{c})$ implies that the Jacobian of $\sigma$ is not vanishing. This Jacobian $J_{\sigma}(\mathbf{u})$ is obtained by taking the determinant $\left|\partial_{\mathbf{u}_{1}} \sigma, \partial_{\mathbf{u}_{2}} \sigma, \partial_{\mathbf{u}_{3}} \sigma\right|$ which expands as

$\sum_{\substack{0 \leq i \leq l_{1}-1, 0 \leq j \leq l_{2}, 0 \leq k \leq l_{3}}} \sum_{\substack{0 \leq i^{\prime} \leq l_{1}, 0 \leq j^{\prime} \leq l_{2}-1,}} \sum_{\substack{0 \leq i^{\prime \prime} \leq l_{1}, 0 \leq k^{\prime \prime} \leq l_{3}, 0 \leq k^{\prime \prime} \leq l_{2},-1}}\left|\Delta_{i, j, k}^{1}, \Delta_{i^{\prime}, j^{\prime}, k^{\prime}}^{2}, \Delta_{i^{\prime \prime}, j^{\prime \prime}, k^{\prime \prime}}^{3}\right| \omega_{i, j, k}^{1} \omega_{i^{\prime}, j^{\prime}, k^{\prime}}^{2} \omega_{i^{\prime \prime}, j^{\prime \prime}, k^{\prime \prime}}^{3} N_{i, j, k}^{1}(\mathbf{u}) N_{i^{\prime}, j^{\prime}, k^{\prime}}^{2}(\mathbf{u}) N_{i^{\prime \prime}, j^{\prime \prime}, k^{\prime \prime}}^{3}(\mathbf{u})$.

As the cones $\mathcal{C}_{1}(\mathbf{c}), \mathcal{C}_{2}(\mathbf{c})$ and $\mathcal{C}_{3}(\mathbf{c})$ are cotransverse, by lemma 1 we deduce that the determinants $\left|\Delta_{i, j, k}^{1}, \Delta_{i^{\prime}, j^{\prime}, k^{\prime}}^{2}, \Delta_{i^{\prime \prime}, j^{\prime \prime}, k^{\prime \prime}}^{3}\right|$ have a constant sign for $\Delta_{i, j, k}^{1} \in \mathcal{C}_{1}(\mathbf{c}), \Delta_{i^{\prime}, j^{\prime}, k^{\prime}}^{2} \in \mathcal{C}_{2}(\mathbf{c})$, $\Delta_{i^{\prime \prime}, j^{\prime \prime}, k^{\prime \prime}}^{3} \in \mathcal{C}_{3}(\mathbf{c})$. As the basis functions and the factors are positive, the Jacobian $J_{\sigma}(\mathbf{u})$ cannot vanish at $\mathbf{u} \in \mathcal{G}$, except if all the $N_{i, j, k}^{1}(\mathbf{u}) N_{i^{\prime}, j^{\prime}, k^{\prime}}^{2}(\mathbf{u}), N_{i^{\prime \prime}, j^{\prime \prime}, k^{\prime \prime}}^{3}(\mathbf{u})$ vanish, which is not possible.

The map $\sigma$ is locally injective on $\mathcal{P}$. By Proposition 2, we deduce that $\sigma$ is globally injective on $\mathcal{P}$. 


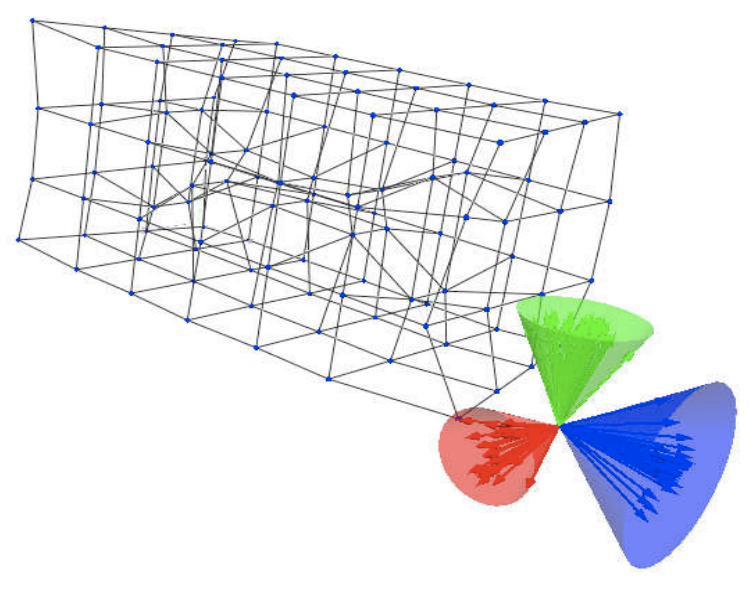

(a) cotransverse cones

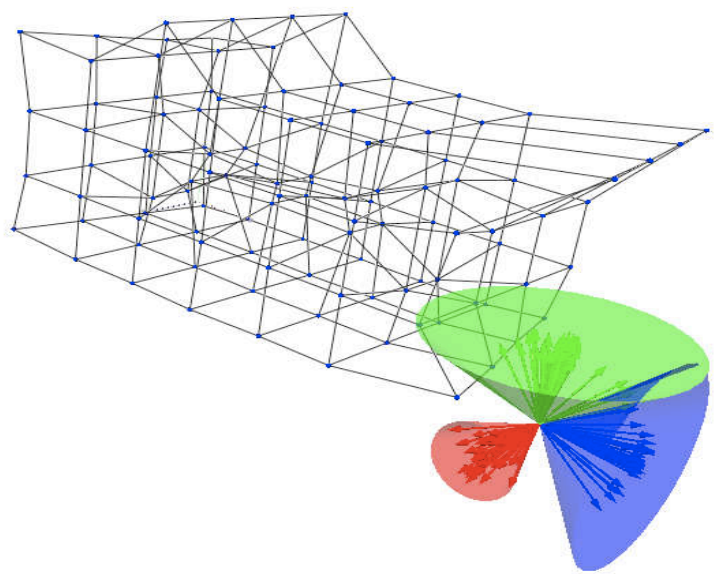

(b) non-cotransverse cones

Fig.1. Injectivity test by cones.

Another type of conditions has been used in [17] or [27] to check the injectivity of a trivariate parametrisation. It involves computatation of circular cones and the analysis of vector products of such circular cones. The one we propose is simple and leads to linear constraints for the interior points as we will see.

Fig.1 shows two examples of the injectivity testing method.

\subsubsection{Linear constraint for injectivity.}

This condition can be used to devise an algorithm which constructs an injective trivariate Bspline parameterization from given boundary control points. Given six boundary surfaces described by the controls points $\mathbf{c}_{i, 0, k}, \mathbf{c}_{i, l_{2}, k}, \mathbf{c}_{0, j, k}, \mathbf{c}_{l_{1}, j, k}, \mathbf{c}_{i, j, 0}, \mathbf{c}_{i, j, l_{3}}$, with $0 \leq i \leq l_{1}, 0 \leq j \leq l_{2}, 0 \leq k \leq l_{3}$, we define the boundary cone $\mathcal{C}_{1}^{0}(\mathbf{c})$ (resp. $\mathcal{C}_{2}^{0}(\mathbf{c}), \mathcal{C}_{3}^{0}(\mathbf{c})$ ) as the cone generated by the vectors $\Delta_{i, j, 0}^{1}(\mathbf{c}), \Delta_{i, j, l_{3}}^{1}(\mathbf{c})$ for $0 \leq i \leq l_{1}-1,0 \leq j \leq l_{2}\left(\operatorname{resp} . \Delta_{0, j, k}^{2}(\mathbf{c}), \Delta_{l_{2}, j, k}^{2}(\mathbf{c})\right.$ for $0 \leq j \leq l_{2}-1,0 \leq$ $k \leq l_{3}, \Delta_{i, 0, k}^{3}(\mathbf{c}), \Delta_{i, l_{2}, k}^{3}(\mathbf{c})$ for $\left.0 \leq i \leq l_{1}, 0 \leq k \leq l_{3}-1\right)$. We assume that these boundary surfaces form a regular boundary and that the three boundary cones $\mathcal{C}_{1}^{0}(\mathbf{c}), \mathcal{C}_{2}^{0}(\mathbf{c}), \mathcal{C}_{3}^{0}(\mathbf{c})$ are cotransverse.

Given six boundary B-spline surfaces, the linear constraint condition for injective trivariate B-spline parameterization can be derived such that the three cones constructed from the first derivative vectors are cotransverse.

As the boundary cones $\mathcal{C}_{1}^{0}(\mathbf{c}), \mathcal{C}_{2}^{0}(\mathbf{c}), \mathcal{C}_{3}^{0}(\mathbf{c})$ are cotransverse, we can find

- a plane $H_{0}$ such that $\forall \mathbf{u} \in \mathcal{C}_{i}^{0, *}(\mathbf{c})(i=1,2,3), H_{0}(\mathbf{u})>0$, and 
- a plane $H_{k}$ such that $\forall \mathbf{u} \in \mathcal{C}_{i}^{0, *}(\mathbf{c}) \cup \mathcal{C}_{j}^{0, *}(\mathbf{c}), H_{k}(\mathbf{u})>0$ and $\forall \mathbf{u} \in \mathcal{C}_{k}^{0, *}(\mathbf{c}), H_{k}(\mathbf{u})<0$ for $\{i, j, k\}=\{1,2,3\}$.

Such separating planes can be deduced easily from convex hull computations of finite sets of vectors, which are generating the cones $\mathcal{C}_{1}^{0}(\mathbf{c}), \mathcal{C}_{2}^{0}(\mathbf{c}), \mathcal{C}_{3}^{0}(\mathbf{c})$ or their unions. Such construction can be considered as the trivariate generalization of the work in [22]. Based on above construction, the linear constraint conditions of inner control points with injective trivariate B-spline parameterization can be presented as follows,

$\left\{\begin{array}{l}H_{0}\left(\mathbf{c}_{i+1, j, k}-\mathbf{c}_{i, j, k}\right)>0, H_{1}\left(\mathbf{c}_{i+1, j, k}-\mathbf{c}_{i, j, k}\right)<0, H_{2}\left(\mathbf{c}_{i+1, j, k}-\mathbf{c}_{i, j, k}\right)>0, H_{3}\left(\mathbf{c}_{i+1, j, k}-\mathbf{c}_{i, j, k}\right)>0, \\ H_{0}\left(\mathbf{c}_{i, j+1, k}-\mathbf{c}_{i, j, k}\right)>0, H_{1}\left(\mathbf{c}_{i, j+1, k}-\mathbf{c}_{i, j, k}\right)>0, H_{2}\left(\mathbf{c}_{i, j+1, k}-\mathbf{c}_{i, j, k}\right)<0, H_{3}\left(\mathbf{c}_{i, j+1, k}-\mathbf{c}_{i, j, k}\right)>0, \\ H_{0}\left(\mathbf{c}_{i, j, k+1}-\mathbf{c}_{i, j, k}\right)>0, H_{1}\left(\mathbf{c}_{i, j, k+1}-\mathbf{c}_{i, j, k}\right)>0, H_{2}\left(\mathbf{c}_{i, j, k+1}-\mathbf{c}_{i, j, k}\right)>0, H_{3}\left(\mathbf{c}_{i, j, k+1}-\mathbf{c}_{i, j, k}\right)<0,\end{array}\right.$ where $0<i<l_{1}, 0 \leq j<l_{2}, 0 \leq k<l_{3}$.

These conditions provide an easy-to-check method for the injectivity of a parameterization. In Section 5, we will employ it to check the injectivity of an initial parameterization.

\section{Isogeometric analysis and parameterization of computational domain}

In this section, we aim at presenting the reasons why solutions from IGA depend strongly on the choice of the parameterization. This will be illustrated on a heat conduction problem.

\subsection{Test model - heat conduction problem}

Given a domain $\Omega$ with $\Gamma=\partial \Omega_{D} \cup \partial \Omega_{N}$, we consider the following thermal conduction problem:

$$
\begin{aligned}
& \boldsymbol{\nabla}(\kappa(\mathbf{x}) \nabla T(\mathbf{x}))=f(\mathbf{x}) \quad \text { in } \Omega \\
& T(\mathbf{x})=T_{0}(\mathbf{x}) \text { on } \partial \Omega_{D} \\
& \kappa(\mathbf{x}) \frac{\partial T}{\partial \mathbf{n}}(\mathbf{x})=\Phi_{0}(\mathbf{x}) \text { on } \partial \Omega_{N}
\end{aligned}
$$

where $\mathbf{x}$ are the Cartesian coordinates, $T$ represents the temperature field and $\kappa$ the thermal conductivity. Dirichlet and Neumann boundary conditions are applied on $\partial \Omega_{D}$ and $\partial \Omega_{N}$ respectively, $T_{0}$ and $\Phi_{0}$ being the imposed temperature and thermal flux (n unit vector normal to the boundary). $f$ is a user-defined function that allows to generate problems with an analytical solution, by adding a source term to the classical heat conduction equation. 


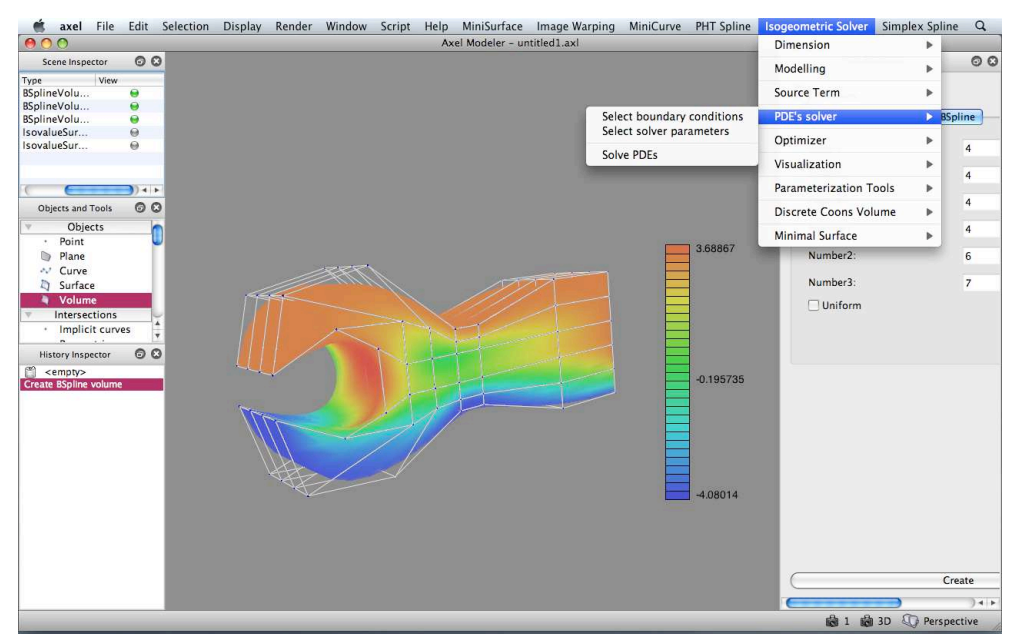

Fig.2. Interface for isogeometric solver in AXEL.

According to a classical variational approach, we seek for a solution $T \in H^{1}(\Omega)$, such as $T(\mathbf{x})=T_{0}(\mathbf{x})$ on $\partial \Omega_{D}$ and:

$$
\int_{\Omega} \boldsymbol{\nabla}(\kappa(\mathbf{x}) \boldsymbol{\nabla} T(\mathbf{x})) \psi(\mathbf{x}) d \Omega=\int_{\Omega} f(\mathbf{x}) \psi(\mathbf{x}) d \Omega \quad \forall \psi \in H_{\partial \Omega_{D}}^{1}(\Omega),
$$

where $\psi(\mathbf{x})$ are test functions. After integrating by parts and using boundary conditions, we obtain:

$$
-\int_{\Omega} \kappa(\mathbf{x}) \nabla T(\mathbf{x}) \nabla \psi(\mathbf{x}) d \Omega+\int_{\partial \Omega_{N}} \Phi_{0}(\mathbf{x}) \psi(\mathbf{x}) d \Gamma=\int_{\Omega} f(\mathbf{x}) \psi(\mathbf{x}) d \Omega .
$$

According to the IGA paradigm, the temperature field is represented using B-spline basis functions. For a 3D problem, we have:

$$
\mathcal{T}(\xi, \eta, \zeta)=\sum_{i=1}^{n_{i}} \sum_{j=1}^{n_{j}} \sum_{k=1}^{n_{k}} \hat{N}_{i}^{p_{i}}(\xi) \hat{N}_{j}^{p_{j}}(\eta) \hat{N}_{k}^{p_{k}}(\zeta) T_{i j k}
$$

where $\hat{N}_{i}$ functions are B-Spline basis functions and $\mathbf{u}=(\xi, \eta, \zeta) \in \mathcal{P}$ are domain parameters. Then, we define the test functions $\psi(\mathbf{x})$ in the physical domain as:

$$
N_{i j k}(\mathbf{x})=\hat{N}_{i j k} \circ \sigma^{-1}(x, y, z)=\hat{N}_{i j k}(\xi, \eta, \zeta)=\hat{N}_{i}^{p_{i}}(\xi) \hat{N}_{j}^{p_{j}}(\eta) \hat{N}_{k}^{p_{k}}(\zeta)
$$

The weak formulation Eq. 2 reads:

$$
\sum_{r=1}^{n_{r}} \sum_{s=1}^{n_{s}} \sum_{t=1}^{t_{l}} T_{r s t} \int_{\Omega} \kappa(\mathbf{x}) \nabla N_{r s t}(\mathbf{x}) \nabla N_{i j k}(\mathbf{x}) d \Omega=\int_{\partial \Omega_{N}} \Phi_{0}(\mathbf{x}) N_{i j k}(\mathbf{x}) d \Gamma-\int_{\Omega} f(\mathbf{x}) N_{i j k}(\mathbf{x}) d \Omega .
$$


Finally, we obtain a linear system similar to that resulting from the classical finite-element methods, with a matrix and a right-hand side defined as:

$$
\begin{aligned}
M_{i j k, r s t} & =\int_{\Omega} \kappa(\mathbf{x}) \nabla N_{r s t}(\mathbf{x}) \nabla_{i j k}(\mathbf{x}) d \Omega \\
& =\int_{\mathcal{P}} \kappa(\mathcal{T}(\mathbf{u})) \nabla_{\mathbf{u}} \hat{N}_{r s t}(\mathbf{u}) B(\mathbf{u})^{T} B(\mathbf{u}) \nabla_{\mathbf{u}} \hat{N}_{r s t}(\mathbf{u}) J(\mathbf{u}) d \mathcal{P} \\
S_{i j k} & =-\int_{\partial \Omega_{N}} \Phi_{0}(\mathbf{x}) N_{i j k}(\mathbf{x}) d \Gamma+\int_{\Omega} f(\mathbf{x}) N_{i j k}(\mathbf{x}) d \Omega \\
& =-\int_{\partial \mathcal{P}_{N}} \Phi_{0}(\mathcal{T}(\mathbf{u})) \hat{N}_{i j k}(\mathbf{u}) J(\mathbf{u}) d \hat{\Gamma}+\int_{\mathcal{P}} f(\mathcal{T}(\mathbf{u})) \hat{N}_{i j k}(\mathbf{u}) J(\mathbf{u}) d \mathcal{P} .
\end{aligned}
$$

where $J$ is the Jacobian of the transformation, $B^{K}$ is the transposed of the inverse of the Jacobian matrix. The above integrations are performed in the parameter space using classical Gauss quadrature rules.

Starting from a trivariate B-spline parametric volume as computational domain, a general framework of an isogeometric solver for heat conduction problem (1) has been implemented as plugins in $\mathrm{AXEL}^{1}$, yielding a B-spline parametric volume with $4 \mathrm{D}$ control points as solution field. This plugin is written in $\mathrm{C}++$ and QT, and has nice user-interface as shown in Fig.2. The visualization tools of analysis results are implemented as a part of the isogeometric toolbox of the project EXCITING ${ }^{2}$.

\subsection{Isogeometric analysis with different parameterization}

As mentioned above, given six boundary B-spline surfaces, we can construct various trivariate B-spline parametric volumes with different parameterizations. For Example I in Fig. 3, we present two kinds of parameterization for a computational domain $\Omega(x, y, z)=[0,6] \times[0,6] \times$ $[0,6]$ represented by cubic B-spline volumes, where the knot vectors in $\xi, \eta$ and $\zeta$ directions are $\{0,0,0,0,1,2,3,4,4,4,4\}$. Fig. 3 (a) and Fig. 3 (b) present two different placements of inner control points, Fig. 3 (c) and Fig. 3 (d) show the isoparametric surfaces on the computational domain with respect to different placements of inner control points.

We test these two parameterizations on the heat conduction problem (1) with source term

$$
\boldsymbol{f}(x, y, z)=-\frac{\pi^{2}}{3} \sin \left(\frac{\pi x}{3}\right) \sin \left(\frac{\pi y}{3}\right) \sin \left(\frac{\pi z}{3}\right) .
$$

\footnotetext{
${ }^{1}$ http://axel.inria.fr/

${ }^{2}$ http://exciting-project.eu/
} 


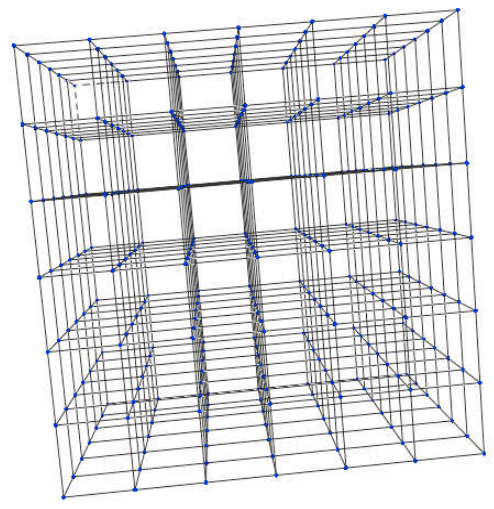

(a) control point placement I

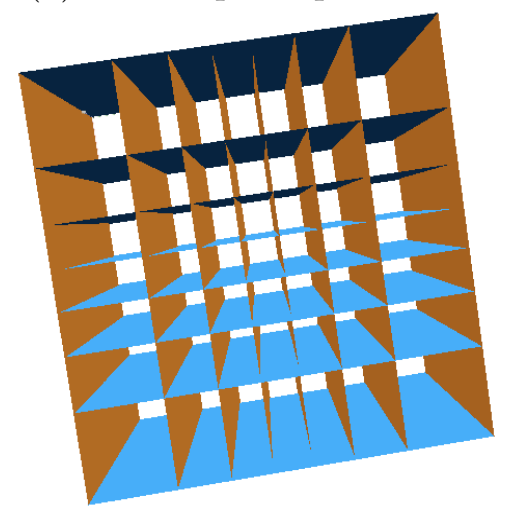

(c) isoparametric surfaces I

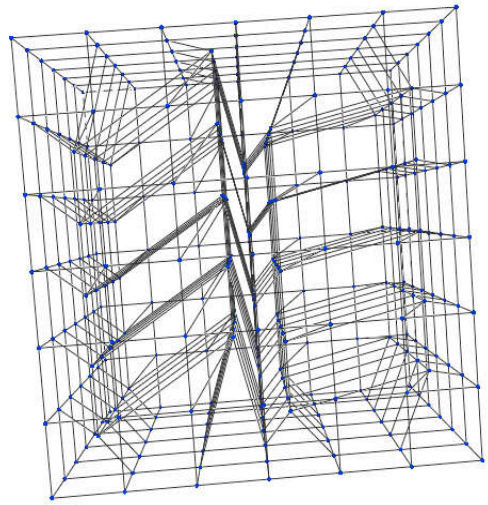

(b) control point placement II

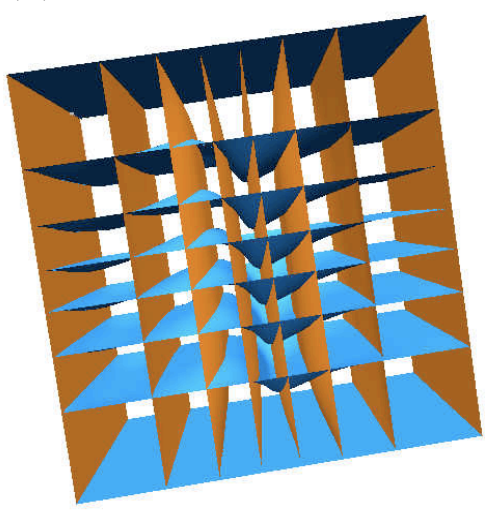

(d) isoparametric surfaces II

Fig.3. Two different parameterizations of computational domains.

For this problem with boundary condition $\boldsymbol{T}_{0}(\boldsymbol{x})=0$ and $\Phi_{0}(\boldsymbol{x})=0$, the exact solution over the computational domain $[0,6] \times[0,6] \times[0,6]$ is

$$
\boldsymbol{T}(x, y, z)=\sin \left(\frac{\pi x}{3}\right) \sin \left(\frac{\pi y}{3}\right) \sin \left(\frac{\pi z}{3}\right) .
$$

Fig.4 (a) show the approximate solution field with respect to parameterization I; Fig.4 (b) show the approximate solution field with respect to parameterization II. In Fig.4 (c), the exact solution field are presented. Obviously, parameterization I is better than parameterization II for this specified heat conduction problem.

Refinement via knot insertion is an efficient operation to improve the result of isogeometric analysis. We compare the error history during refinement operation for these two different param- 


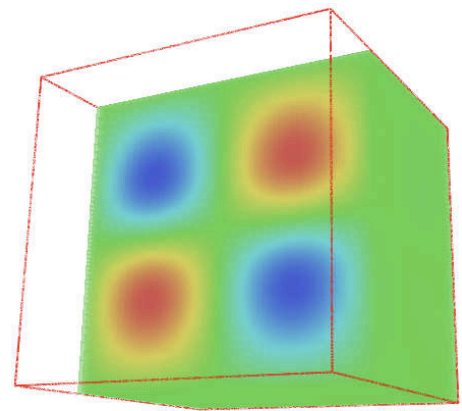

(a)

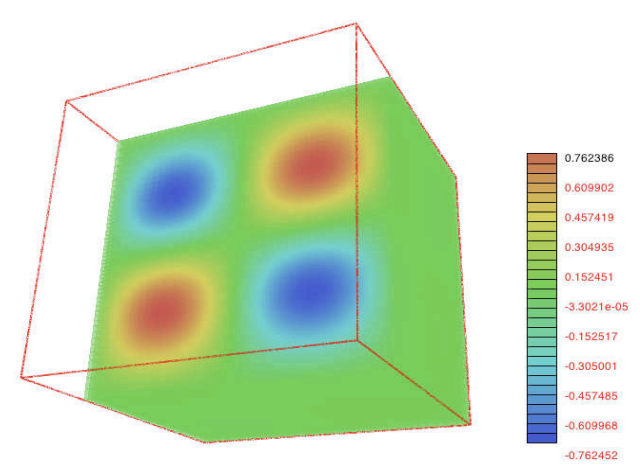

(c)

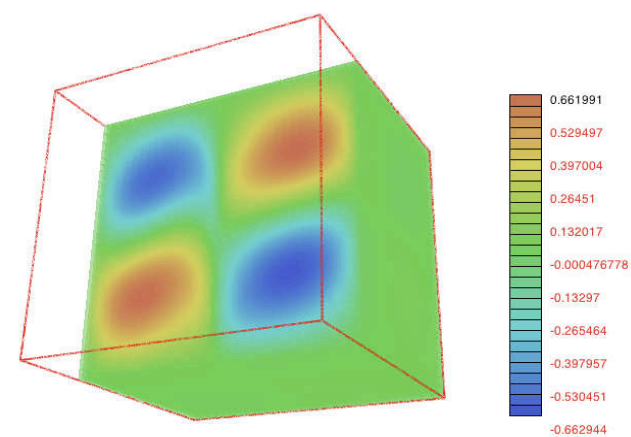

(b)

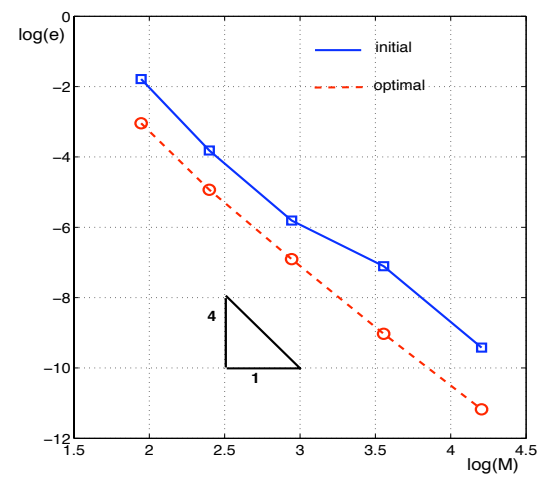

(d)

Fig.4. Simulation results and exact solution: (a). solution field of parameterization I ; (b). solution field of parameterization II ; (c). exact solution field; (d). error analysis with the curve $(\log M, \log e)$, where $M$ is the cube root of number of control points in each refinement. 
eterization in Fig.4 (d). The error is computed in relative $L_{2}$ norm as follows [24]

$$
e=\sqrt{\frac{\int_{\Omega}(\boldsymbol{T}-\tilde{\boldsymbol{T}})^{T}(\boldsymbol{T}-\tilde{\boldsymbol{T}}) d \Omega}{\int_{\Omega} \boldsymbol{T}^{T} \boldsymbol{T} d \Omega}},
$$

where $\boldsymbol{T}$ is the exact solution and $\tilde{\boldsymbol{T}}$ is the approximate solution. From Fig.4, we see that different parameterizations have different impact osn the final result after refinement operation. Though the convergence rates of the two different parameterization are in good agreement with theoretical convergence ( 4 for cubic parameterization), for an error value about $4 \times 10^{-5}$, parameterization I requires $19 \times 19 \times 19$ control points, and parameterization II requires $35 \times 35 \times 35$ control points.

The above example and its analysis show that good parameterization of computational domain is a key issue for IGA. In the next section, we will propose a shape optimization method to construct optimal parameterization of a computational domain.

\section{Optimization method for parametrization of computational domain}

\subsection{Problem statement}

The problem studied in this section can be stated as follows: given six boundary B-spline surfaces, find the inner control points such that the trivariate B-spline parameterization of a computational domain is optimal for an IGA problem with known exact solution. The extension of the proposed method to isogeometric problems without known exact solution is one of our ongoing work.

\subsection{Shape optimization method}

The shape optimization problem consists in finding the shape which is optimal in that it minimizes a certain cost function while satisfying given constraints. The purpose of shape optimization in $\mathrm{CAE}$ is to optimize the CAD object for some physical problem, and the design variables are the control points of the CAD object. For 3D isogeometric shape optimization problems, the design variables are the control points of boundary B-spline surfaces.

Inspired from the idea of shape optimization, in order to obtain optimal parameterization of computational domain, we should let the inner control points, rather than boundary control points, be the design variables for the shape optimization, and find the best placement of inner control points to make the value of a cost function as small as possible. 


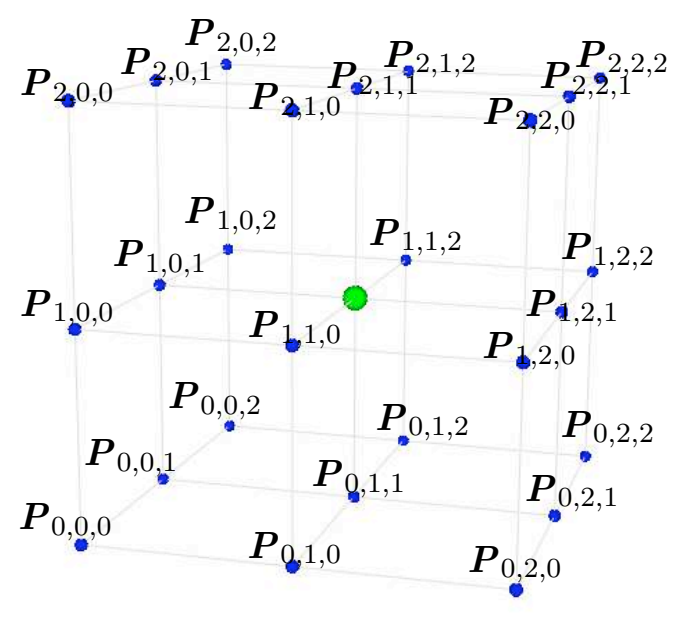

Fig.5. Boundary control points for $l=m=n=2$.

\subsubsection{Initial construction of inner control points.}

For the shape optimization problem, we need to construct an initial placement of inner control points as starting point in the iteration process. In this paper, we propose discrete Coons method for parametric volume generation from given boundary surfaces, which is a trivariate generalization of the method proposed in [16]. For B-spline volume generation, the inner control points can be obtained by the linear combination of the boundary control points.

Suppose that given boundary surfaces are B-spline surfaces, the opposite boundary B-spline surfaces have the same degree, number of control points and knot vectors. If the boundary control points are given as shown in Fig.5, then the interior control points $\boldsymbol{P}_{i, j, k}$ can be constructed as 
follows,

$$
\begin{aligned}
\boldsymbol{P}_{i, j, k}= & (1-i / l) \boldsymbol{P}_{0, j, k}+i / l \boldsymbol{P}_{l, j, k}+(1-j / m) \boldsymbol{P}_{i, 0, k}+j / m \boldsymbol{P}_{i, m, k} \\
& +(1-k / n) \boldsymbol{P}_{i, j, 0}+k / n \boldsymbol{P}_{i, j, n}-[1-i / l, i / l]\left[\begin{array}{cc}
\boldsymbol{P}_{0,0, k} & \boldsymbol{P}_{0, m, k} \\
\boldsymbol{P}_{l, 0, k} & \boldsymbol{P}_{l, m, k}
\end{array}\right]\left[\begin{array}{c}
1-j / m \\
j / m
\end{array}\right] \\
& -[1-j / m, j / m]\left[\begin{array}{rr}
\boldsymbol{P}_{i, 0,0} & \boldsymbol{P}_{i, 0, n} \\
\boldsymbol{P}_{i, m, 0} & \boldsymbol{P}_{i, m, n}
\end{array}\right]\left[\begin{array}{c}
1-k / n \\
k / n
\end{array}\right] \\
& -[1-k / n, k / n]\left[\begin{array}{rr}
\boldsymbol{P}_{0, j, 0} & \boldsymbol{P}_{l, j, 0} \\
\boldsymbol{P}_{0, j, n} & \boldsymbol{P}_{l, j, n}
\end{array}\right]\left[\begin{array}{c}
1-i / l \\
i / l
\end{array}\right] \\
& \left.+(1-k / n)[1-i / l, i / l]\left[\begin{array}{rr}
\boldsymbol{P}_{0,0,0} & \boldsymbol{P}_{0, m, 0} \\
\boldsymbol{P}_{l, 0,0} & \boldsymbol{P}_{l, m, 0}
\end{array}\right]\left[\begin{array}{c}
1-j / m \\
j / m
\end{array}\right]\right] \\
& \left.+k / n[1-i / l, i / l]\left[\begin{array}{rr}
\boldsymbol{P}_{0,0, n} & \boldsymbol{P}_{0, m, n} \\
\boldsymbol{P}_{l, 0, n} & \boldsymbol{P}_{l, m, n}
\end{array}\right]\left[\begin{array}{c}
1-j / m \\
j / m
\end{array}\right]\right]
\end{aligned}
$$

Then the corresponding B-spline volume has the following form

$$
\sigma(\xi, \eta, \zeta)=\sum_{i=0}^{l} \sum_{j=0}^{m} \sum_{k=0}^{k} \boldsymbol{P}_{i, j, k} N_{i}(\xi) N_{j}(\eta) N_{k}(\zeta) .
$$

where $N_{i}(\xi), N_{j}(\eta)$ and $N_{k}(\zeta)$ are B-spline basis function with knot vectors given by boundary surfaces. Fig. 6 presents an example of resulted Coons volume by this method.

Remarks 2. Since the sum of the coefficients equals 1, the resulting inner control points lie in the convex hull of the boundary control points.

Remarks 3. For some given boundary surfaces, this construction may cause some self-intersections, and lead to an improper parameterization for IGA. We use the linear injectivity condition proposed in Section 3 to check the injectivity of initial parameterization. If it does not satisfy the condition, the linear programming method is used to produce another initial parameterization.

\subsubsection{Optimization method.}

In the proposed approach, we minimize the error computed from the IGA solution and the exact solution, by moving inner control points of the computational domain. Therefore, we consider the coordinates of the inner control points as optimization variables and the error of the IGA solution as cost function. The optimization algorithm used for this study is a classical steepest-descent 


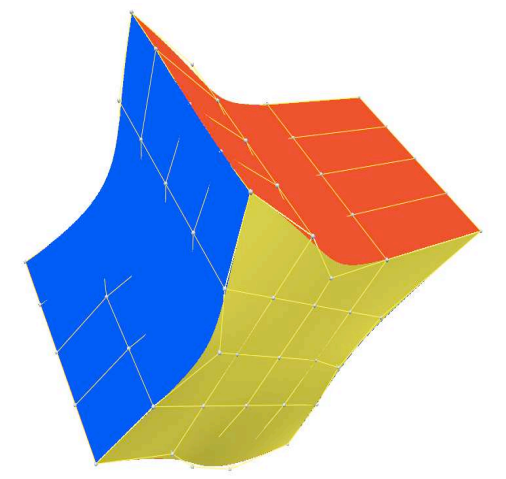

(a) Boundary B-spline surfaces

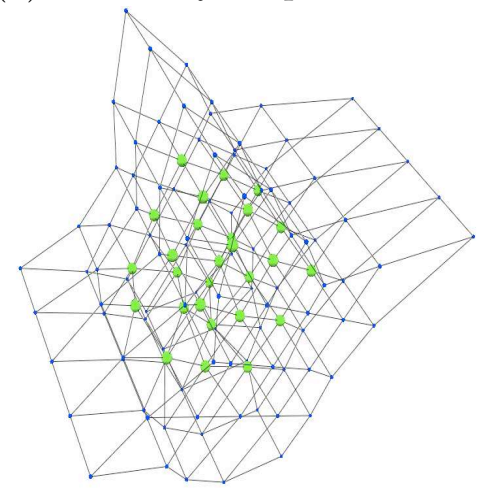

(c) B-spline control lattice

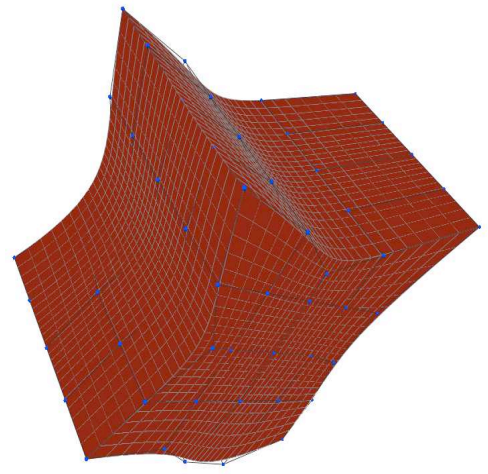

(b) B-spline Coons volume

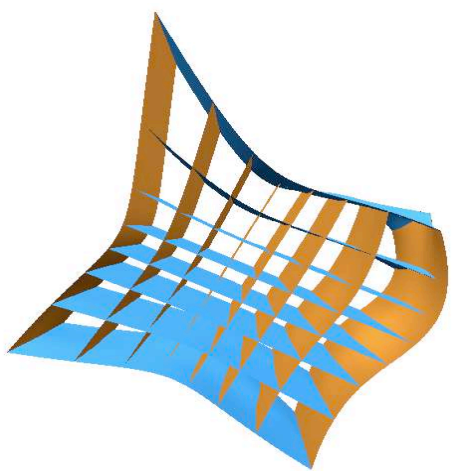

(c) Iso-parametric surfaces

Fig.6. B-spline Coons volume.

method in conjunction with a back-tracking line-search. For this exercise, the gradient of the cost function is approximated using a centered finite-differencing scheme.

Each iteration $k$ of the optimization algorithm can be summarized as follows, starting from a point $x_{k}$ in the variable space, we perform the following operations:

1. Evaluation of perturbed points $x_{k}+\epsilon e_{k}$;

2. Estimation of the gradient $\nabla f\left(x_{k}\right)$ by finite-difference;

3. Define search direction $d_{k}=-\nabla f\left(x_{k}\right)$;

4. Line search : find $\rho$ such as $f\left(x_{k}+\rho d_{k}\right)<f\left(x_{k}\right)$;

These steps are carried out until a stopping criterion is satisfied.

\subsection{Examples and comparison}

In this section, we will present some parameterization results and compare them with the initial solution with respect to the heat conduction problem (1). 


\subsubsection{Example II .}

The second example is for the parameterization of the domain $\Omega=[0,3] \times[0,3] \times[0,3]$ by cubic Bézier surfaces. The corresponding source term and exact solution is presented in (3) and (4). The parameterization result and comparison with initial parameterization are shown in Fig.7. The initial error is reduced by $20.34 \%$ as shown in Fig.7 (e). The final parameterization is clearly better than the initial parameterization during refinement operations as presented in Fig.7 (f).

\subsubsection{Example III .}

The next example is for the parameterization of the domain

$$
\Omega(x, y, z)=\left\{(x, y, z) \mid-1 \leq y \leq x^{2}, 0 \leq x \leq 1,0 \leq z \leq 1\right\}
$$

by trivariate Bézier volume with degree $3 \times 6 \times 3$. The parabolic ruled surface is represented by degenerate bicubic Bézier surface. For the problem with boundary condition $\boldsymbol{T}_{0}(\boldsymbol{x})=0$ and $\Phi_{0}(\boldsymbol{x})=0$ in (1), we can construct an exact solution $\boldsymbol{T}(x, y, z)$ as follows

$$
\boldsymbol{T}(x, y, z)=\sin \left(\pi\left(y-x^{2}\right)\right) \sin (\pi x) \sin (\pi y) \sin (\pi z) .
$$

The initial placement of inner control points is produced by the discrete Coons method as shown in Fig.8 (a). The final parameterization results and some comparisons are also shown in Fig.8. We can find that there are some self-intersections on the control lattice in Fig.8 (b). However, there is no self-intersection on the final parameterization as shown in Fig.8 (c). During the optimization, the initial error is reduced by $6.61 \%$ as shown in Fig.8 (g). The error history during refinement operation is presented in Fig. $8(\mathrm{~h})$. For an error value about $3 \times 10^{-4}$, initial parameterization requires $5 \times 5 \times 11$ control points, and final parameterization requires $11 \times 11 \times 35$ control points.

\subsubsection{Example $I V$.}

The final example is for the parameterization of the domain $\Omega=[0,6] \times \times[0,3] \times[0,3]$ by cubic B-spline surface with knot vector $\{0,0,0,0,1,2,3,4,4,4,4\}$ in the $\xi$ direction and knot vector $\{0,0,0,0,1,1,1,1\}$ in the $\eta$ and $\zeta$ directions. The corresponding source term and exact solution is presented in (3) and (4). The initial placement of inner control points is non-uniform as shown in Fig.9 (a), and the final parametrization result and some comparison are also shown in Fig.9. During the optimization, the initial error is reduced by $2.34 \%$ as shown in Fig.9 (g). The error history during refinement operation is presented in Fig.9 (h). 


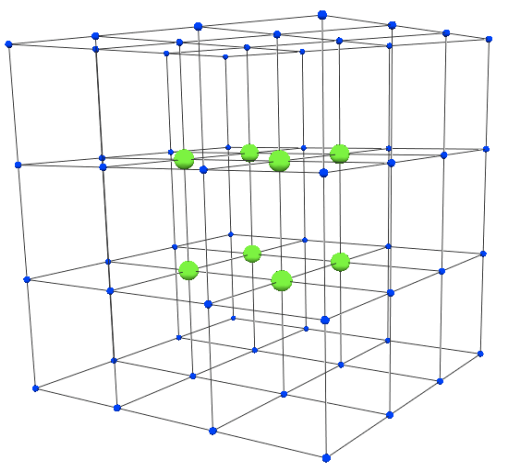

(a) initial control lattice

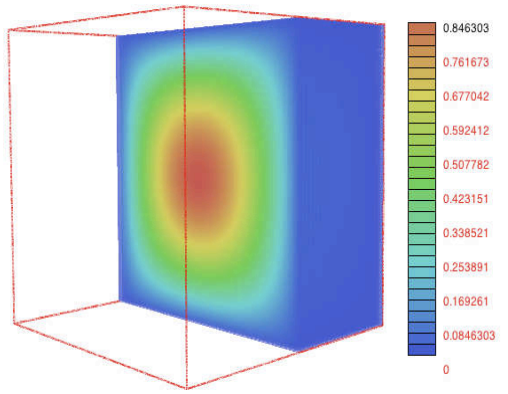

(c) final solution field

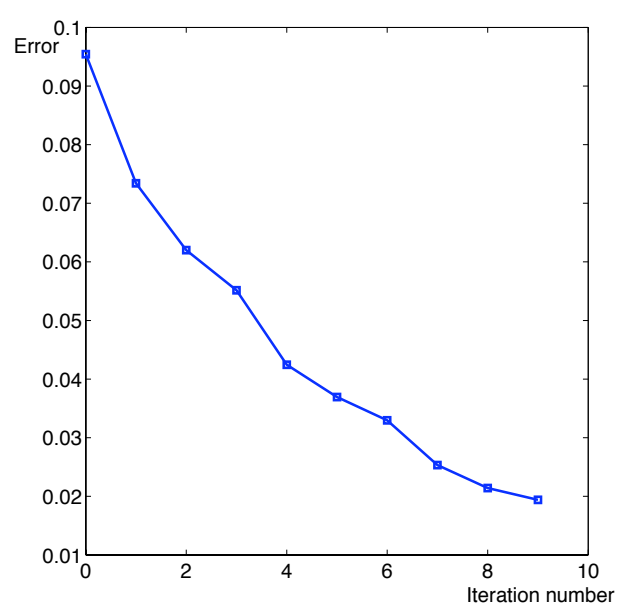

(e) error history during the optimization process

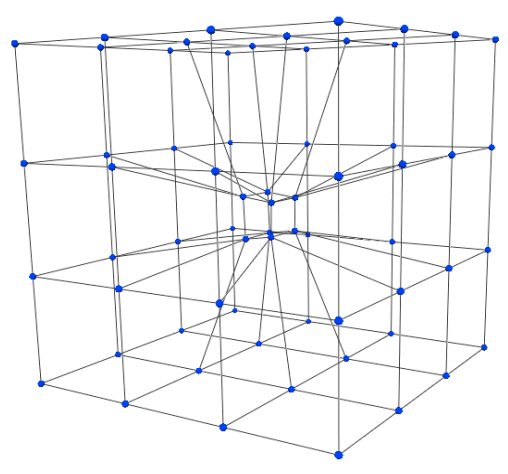

(b) final control lattice

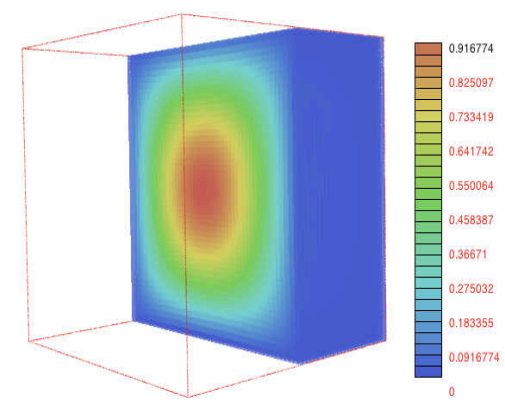

(d) exact solution field

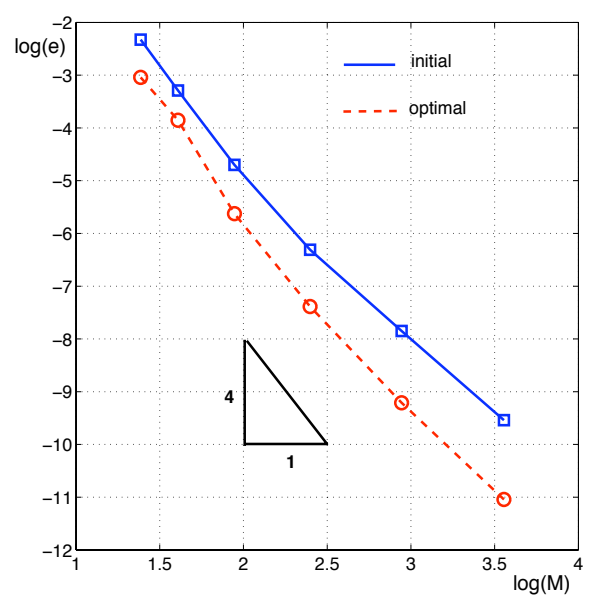

(f) error history during the refinement process

Fig.7. Example II. 


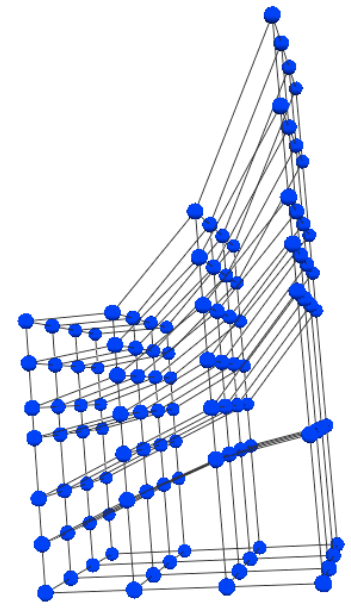

(a) initial control lattice
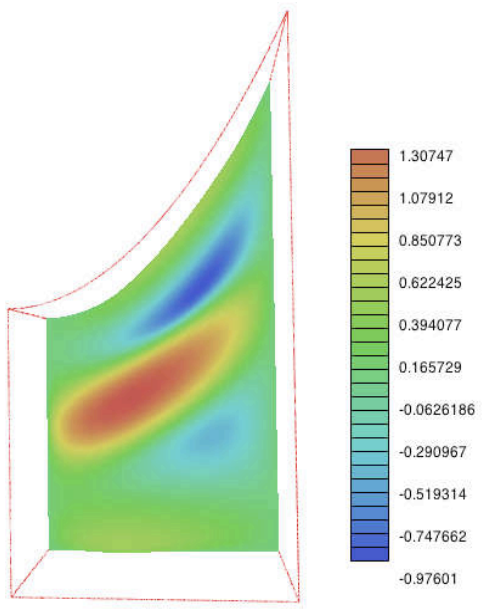

(d) initial solution field

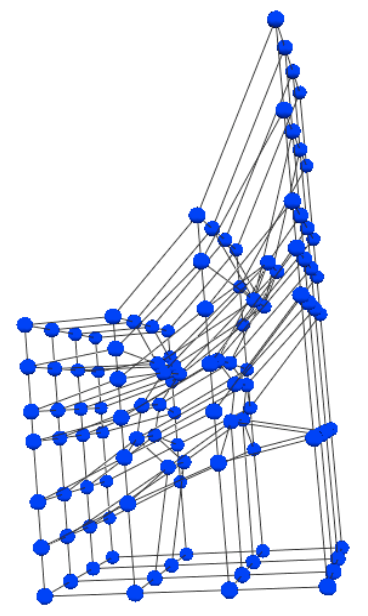

(b) final control lattice

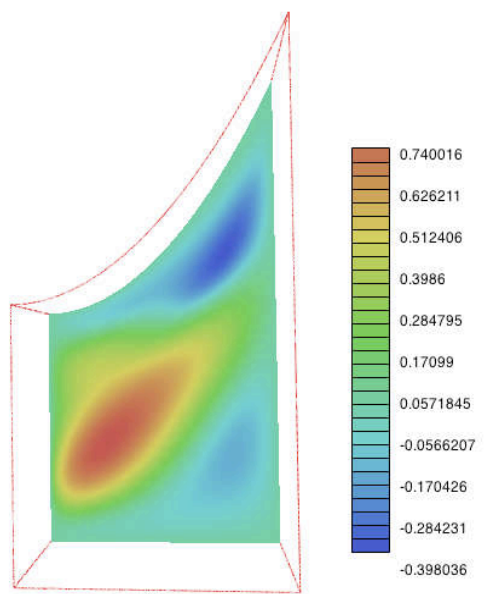

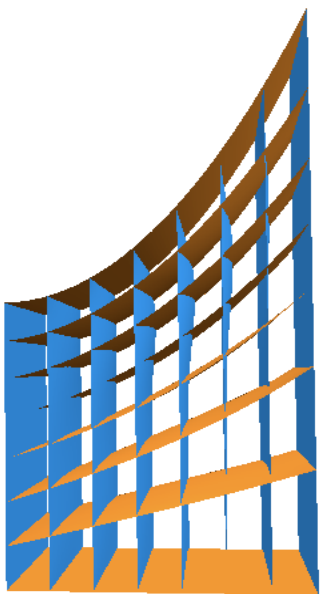

(c) final iso-parametric surfaces

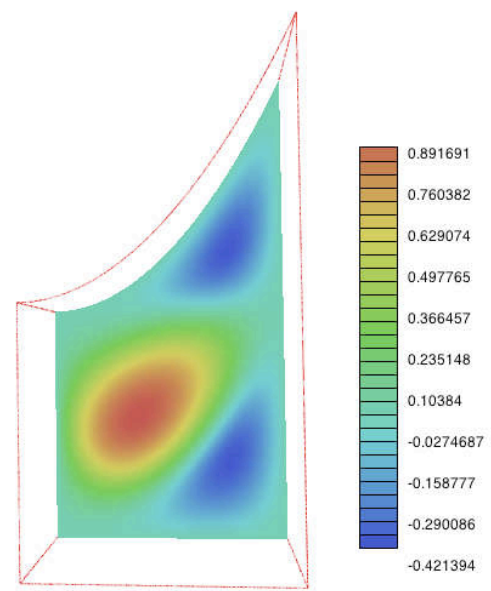

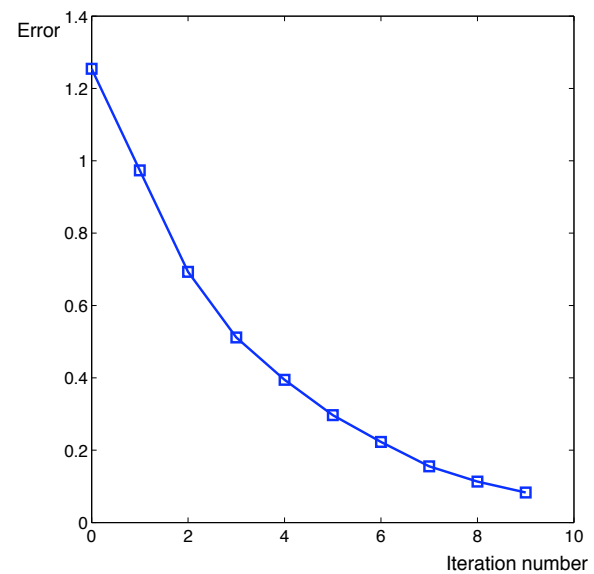

(g) error history during the optimization (e) final solution field

(f) exact solution field

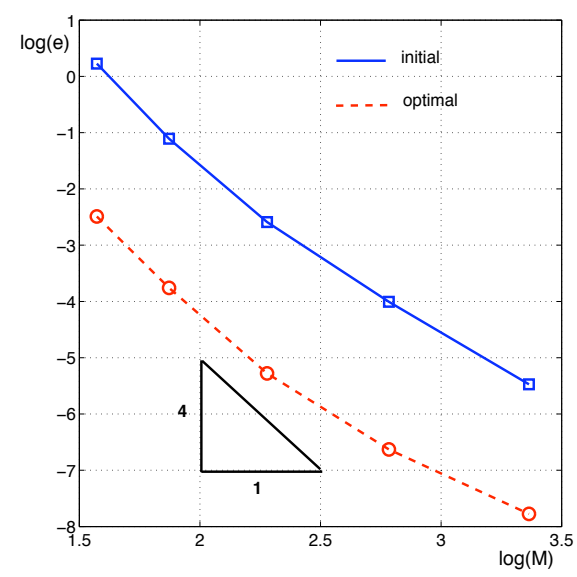

(h) error history during the refinement process

Fig.8. Example III. 


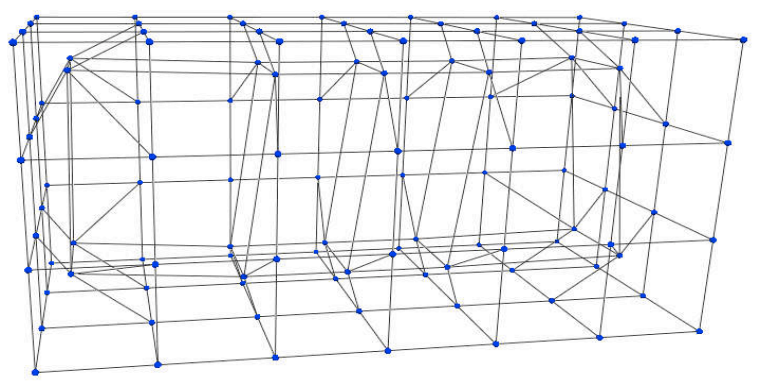

(a) initial control lattice

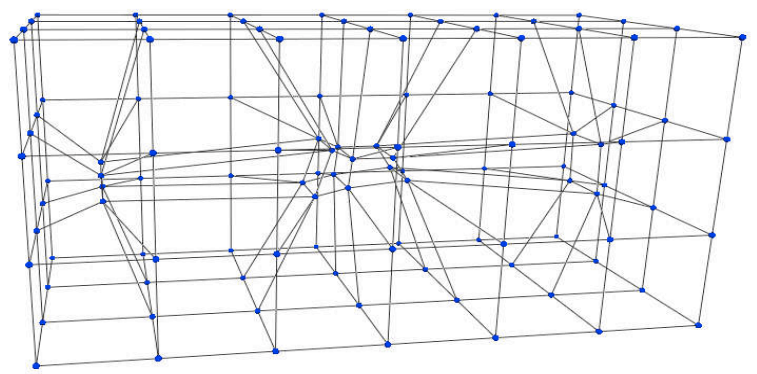

(c) final control lattice

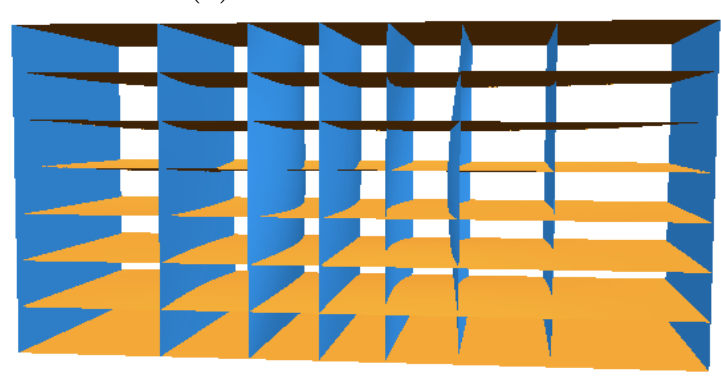

(e) final iso-parametric surfaces

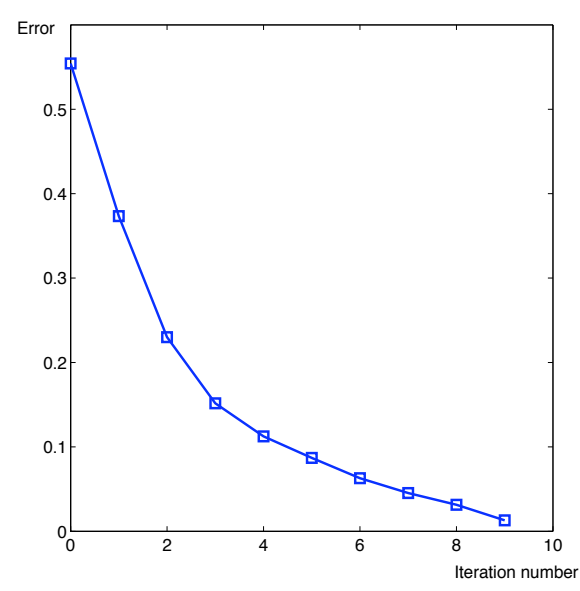

(g) error history during the optimization

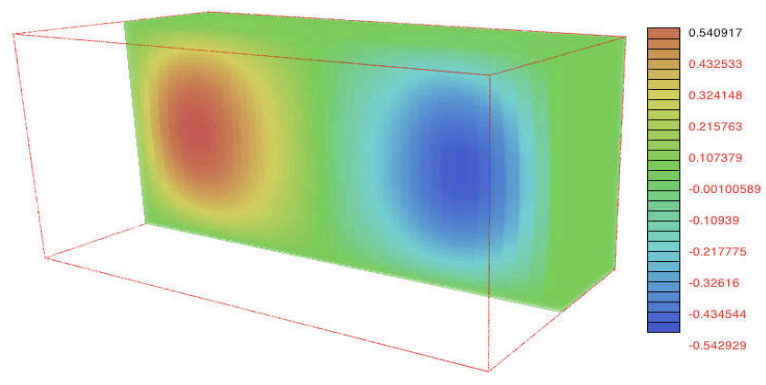

(b) initial solution field

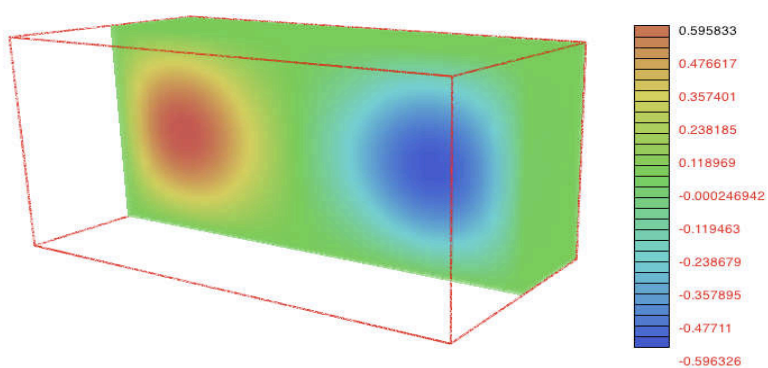

(d) final solution field

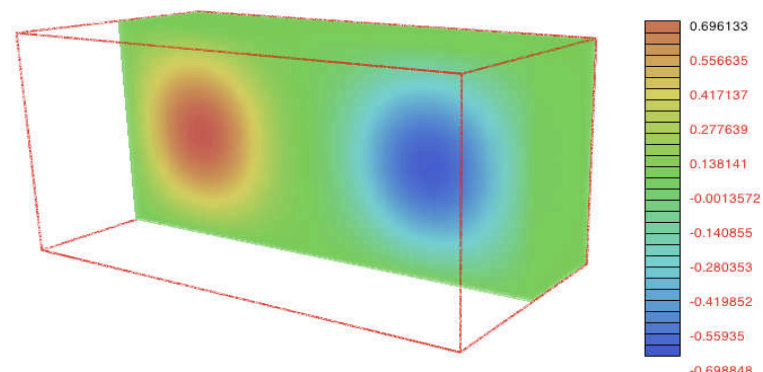

(f) exact solution field

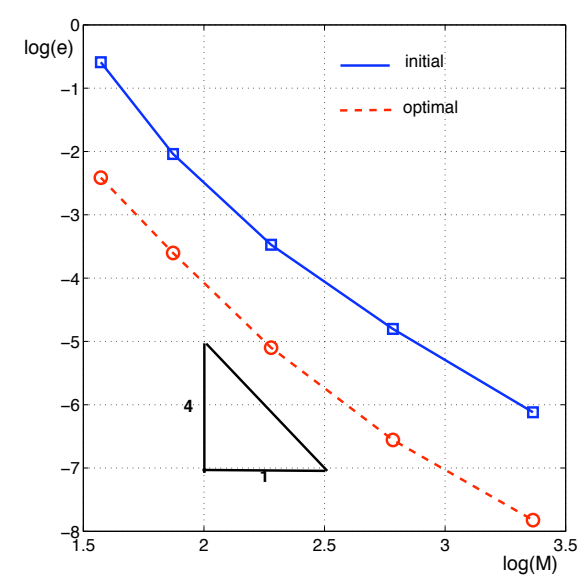

(h) error history during the refinement process

Fig.9. Example IV. 


\section{Conclusion and future work}

Parameterization of computational domains is the first step in an IGA process. In this paper, we show that for different parameterizations of a 3D computational domain, significantly different simulation results can be obtained. Based on this observation and inspired by shape optimization, an approach for optimal parameterization of 3D computational domain is proposed for problems with exact solution. We also proposed a linear and easy-to-check sufficient condition for injectivity of trivariate B-spline parameterization, and a B-spline parametric volume construction method from given boundary surfaces. Several examples are presented to illustrate the effectiveness of the proposed method.

The construction of an optimal parameterization of 3D computational domain for general problem, in which the exact solution is unknown, is also a part of our ongoing work. One possible way is to find a proper a posteriori error estimation method for IGA, and perform the optimization based on this estimation. We will discuss this topic in another paper.

\section{Acknowledgments.}

The authors are supported by the 7th Framework Program of the European Union, project SCP8-218536 "EXCITING". The first author is partially supported by the National Nature Science Foundation of China (No.61004117) and the Zhejiang Provincial Natural Science Foundation of China under Grant No. Y1090718.

\section{References}

[1] M. Aigner, C. Heinrich, B. Jüttler, E. Pilgerstorfer, B. Simeon and A.-V. Vuong. Swept volume parametrization for isogeometric analysis. In E. Hancock and R. Martin (eds.), The Mathematics of Surfaces (MoS XIII 2009), LNCS vol. 5654(2009), Springer, pp.19-44

[2] F. Auricchio, L.B. da Veiga, A. Buffa, C. Lovadina, A. Reali, and G. Sangalli. A fully locking-free isogeometric approach for plane linear elasticity problems: A stream function formulation. Computer Methods in Applied Mechanics and Engineering, 197:160-172, 2007.

[3] Y. Bazilevs, L. Beirao de Veiga, J.A. Cottrell, T.J.R. Hughes, and G. Sangalli. Isogeometric analysis: approximation, stability and error estimates for refined meshes. Mathematical Models and Methods in Applied Sciences, 6:1031-1090, 2006.

[4] Y. Bazilevs, V.M. Calo, T.J.R. Hughes, and Y. Zhang. Isogeometric fluid structure interaction: Theory, algorithms, and computations. Computational Mechanics, 43:3-37, 2008. 
[5] Y. Bazilevs, V.M. Calo, Y. Zhang, and T.J.R. Hughes. Isogeometric fluid structure interaction analysis with applications to arterial blood flow. Computational Mechanics, 38:310-322, 2006.

[6] Y. Bazilevs and T.J.R. Hughes. NURBS-based isogeometric analysis for the computation of flows about rotating components. Computational Mechanics, 43:143-150, 2008.

[7] Y. Bazilevs, V.M. Calo, J.A. Cottrell, J. Evans, T.J.R. Hughes, S. Lipton, M.A. Scott, and T.W. Sederberg. Isogeometric analysis using T-Splines. Computer Methods in Applied Mechanics and Engineering, 199(5-8): 229-263, 2010.

[8] D. Burkhart, B. Hamann and G. Umlauf. Iso-geometric analysis based on Catmull-Clark subdivision solids. Computer Graphics Forum, 29(5): 1575-1584, 2010.

[9] E. Cohen, T. Martin, R.M. Kirby, T. Lyche and R.F. Riesenfeld, Analysis-aware Modeling: Understanding Quality Considerations in Modeling for Isogeometric Analysis. Computer Methods in Applied Mechanics and Engineering, 199(5-8): 334-356, 2010.

[10] J.A. Cottrell, T.J.R. Hughes, and A. Reali. Studies of refinement and continuity in isogeometric analysis. Computer Methods in Applied Mechanics and Engineering, 196:4160-4183, 2007.

[11] J.A. Cottrell, A. Reali, Y. Bazilevs, and T.J.R. Hughes. Isogeometric analysis of structural vibrations. Computer Methods in Applied Mechanics and Engineering, 195:5257-5296, 2006.

[12] T. Dokken, V. Skytt, J. Haenisch, K. Bengtsson. Isogeometric representation and analysis-bridging the gap between CAD and analysis. 47th AIAA Aerospace Sciences Meeting including The New Horizons Forum and Aerospace Exposition, 5-8 January 2009, Orlando, Florida.

[13] M. Dörfel, B. Jüttler, and B. Simeon. Adaptive isogeometric analysis by local h-refinement with T-splines. Computer Methods in Applied Mechanics and Engineering, 199(5-8): 264-275, 2010.

[14] T. Elguedj, Y. Bazilevs, V.M. Calo, and T.J.R. Hughes. $\bar{B}$ and $\bar{F}$ projection methods for nearly incompressible linear and non-linear elasticity and plasticity using higher-order NURBS elements. Computer methods in applied mechanics and engineering, 197: 2732-2762,2008.

[15] J.A. Evans, Y. Bazilevs, I. Babuka, T.J.R. Hughes. $n$-Widths, supinfs, and optimality ratios for the k-version of the isogeometric finite element method, Computer Methods in Applied Mechanics and Engineering, 198: 1726$1741,2009$.

[16] Gerald Farin, Dianne Hansford. Discrete coons patches. Computer Aided Geometric Design, 16(7):691-700, 1999.

[17] James E. Gain, Neil A. Dodgson. Preventing self-Intersection under free-form deformation. IEEE Transactions on Visualization and Computer Graphics, 7(4), pp. 289-298, Oct.-Dec. 2001.

[18] H. Gomez, V.M. Calo, Y. Bazilevs, and T.J.R. Hughes. Isogeometric analysis of the Cahn-Hilliard phase-field model. Computer Methods in Applied Mechanics and Engineering, 197:4333-4352, 2008.

[19] T.J.R. Hughes, J.A. Cottrell, Y. Bazilevs. Isogeometric analysis: CAD, finite elements, NURBS, exact geometry, and mesh refinement. Computer Methods in Applied Mechanics and Engineering 194, 39-41, pp 4135-4195, 2005.

[20] T.J.R. Hughes, A. Realli, and G. Sangalli. Duality and unified analysis of discrete approximations in structural dynamics and wave propagation: Comparison of p-method finite elements with k-method NURBS. Computer methods in applied mechanics and engineering, 197: 4104-4124, 2008. 
[21] T.J.R. Hughes, A. Realli, and G. Sangalli. Efficient quadrature for NURBS-based isogeometric analysis. Computer Methods in Applied Mechanics and Engineering, 199(5-8): 301-313, 2010.

[22] B. Jüttler. Shape-preserving least-squares approximation by polynomial parametric spline curves. Computer Aided Geometric Design, 14: 731-747, 1997.

[23] H. Kestelman. Mappings with non-vanishing Jacobian. Amer. Math. Monthly 78, 662-663, 1971.

[24] H.J. Kim, Y.D Seo and S.K Youn. Isogeometric analysis for trimmed CAD surfaces. Computer Methods in Applied Mechanics and Engineering, 198(37-40): 2982-2995, 2009.

[25] T. Martin, E. Cohen, and R.M. Kirby. Volumetric parameterization and trivariate B-spline fitting using harmonic functions. Computer Aided Geometric Design, 26(6):648-664, 2009.

[26] Thien T. Nguyen and B. Jüttler. Using approximate implicitization for domain parameterization in isogeometric analysis. International Conference on Curves and Surfaces, Avignon, France, 2010.

[27] Thomas W. Sederberg, Ray J. Meyers. Loop detection in surface patch intersections, Computer Aided Geometric Design, 5:2, 161 - 171, 1988.

[28] R. Sevilla, S. Fernandes-Mendez, and A. Huerta. NURBS-enhanced finite element method for Euler equations. International Journal for Numerical Methods in Fluids, 57: 1051-1069, 2008.

[29] W.A. Wall, M.A. Frenzel, and C. Cyron. Isogeometric structural shape optimization. Computer Methods in Applied Mechanics and Engineering, 197:2976-2988, 2008.

[30] G. Xu, B. Mourrain, R. Duvigneau and A. Galligo. Optimal analysis-aware parameterization of computational domain in isogeometric analysis. Proc. of Geometric Modeling and Processing (GMP 2010), 2010, 236-254

[31] Y. Zhang, Y. Bazilevs, S. Goswami, C. Bajaj, and T.J.R. Hughes. Patient-specific vascular NURBS modeling for isogeometric analysis of blood flow. Computer Methods in Applied Mechanics and Engineering, 196:2943-2959, 2007. 